\title{
Lipid Peroxidation and Antioxidant Status in Nonobese Type 2 Diabetes Mellitus
}

\author{
Vineet Kumar Khemka, ${ }^{1}$ Subhadip Choudhuri, ${ }^{1}$ Anirban Ganguly, \\ Arindam Ghosh, ${ }^{1}$ Aritri Bir, ${ }^{1,2}$ and Anindita Banerjee ${ }^{1,2}$ \\ ${ }^{1}$ Department of Biochemistry, Institute of Post Graduate Medical Education and Research, Kolkata 700020, India \\ ${ }^{2}$ Department of Biochemistry, ICARE Institute of Medical Sciences and Research, Banabishnupur, Haldia 721645, India
}

Correspondence should be addressed to Anindita Banerjee; anny.banerjee@gmail.com

Received 30 May 2014; Revised 13 August 2014; Accepted 26 September 2014; Published 22 October 2014

Academic Editor: Sarantis Livadas

Copyright ( 2014 Vineet Kumar Khemka et al. This is an open access article distributed under the Creative Commons Attribution License, which permits unrestricted use, distribution, and reproduction in any medium, provided the original work is properly cited.

\begin{abstract}
The aim of the study was to investigate the association between lipid peroxidation and antioxidant status in nonobese type 2 diabetes mellitus (T2DM) and further to correlate whether their significant association is putatively associated with the pathogenesis of T2DM. A number of 102 nonobese T2DM subjects and 95 nondiabetic subjects as healthy controls were enrolled in this crosssectional study. Serum samples were collected in cryovials for malondialdehyde (MDA) and thiol assays. Total thiol or sulfhydryl $(-\mathrm{SH})$ groups in peripheral blood mononuclear cells (PBMCs) and sera, as well as level of MDA, a marker for lipid peroxidation in serum, were measured spectrophotometrically. Serum MDA level was found significantly higher whereas serum and PBMC total thiol levels were diminished significantly among nonobese T2DM subjects compared to HC subjects. Moreover, serum MDA level is found to have a significant inverse correlation with serum total thiol and PBMC thiol levels among DM subjects, but no significant correlation was observed in HC individuals. A significant inverse correlation between serum MDA and serum total thiol levels among nonobese T2DM subjects suggests a close association of increased oxidative stress with decreased antioxidant status in nonobese T2DM.
\end{abstract}

\section{Introduction}

Diabetes mellitus (DM), the most common metabolic disorder worldwide and one of the leading causes of death in developed countries, is considered as a major threat to human health in the 21st century. Although the pancreatic beta-cell and its secretory product insulin are central in the pathophysiology of DM, the pathogenic mechanisms by which hyperglycemia appears differ widely. Insulin is the principal hormone that regulates uptake of glucose from the blood into most cells (primarily muscle and fat cells, but not central nervous system cells). Thus, deficiency of insulin (type 1) or the insensitivity of insulin receptors or both together (type 2) play a pivotal role in all forms of DM [1-3]. Although the causes of type $2 \mathrm{DM}$ are multifactorial and include both genetic and environmental elements affecting beta-cell functions and tissue insulin sensitivity (muscle, liver, adipose tissue, pancreas, etc.), pancreatic beta-cell dysfunction and reduced insulin sensitivity are believed to play a key role in the pathogenesis of type $2 \mathrm{DM}[3,4]$.

Different studies suggest that metabolic dysregulation through oxidative stress might have an important role in the pathogenesis of DM [5]. Uncoupling of intracellular NADPH oxidase, the pivotal signaling cascade, results in the increased generation of intracellular reactive oxygen species (ROS). Under chronic hyperglycemic state, oxidative stress develops in diabetes due to poor elimination of ROS from different tissues [6, 7]. It has been postulated that the possible cause for this systemic prooxidant and antioxidant imbalance may be due to diminished activity of antioxidant defense system which is normally responsible for scavenging free radicals from the cells. Oxidation of complex lipids in vivo is largely caused by ROS such as hydroxyl radical, which in turn are synthesized by lipoxygenases as a response to cell injury, typically from hydrogen peroxide. The major targets of these damaging species are the long-chain polyunsaturated 
fatty acids of cellular phospholipids, which are particularly susceptible to attack because of the arrangement of double and single bonds. The resultant lipid peroxide frequently decomposes to a radical, which further reacts with the most biologically important molecules like proteins and lipids. Further decomposition of these lipid peroxides produces toxic malondialdehyde (MDA), formed particularly from arachidonic acid [8]. However, total thiols $(-\mathrm{SH})$ that exist extra- as well as intracellularly in free (reduced glutathione) or bound (protein-bound) forms reduce highly reactive superoxide radicals, and thus maintain the intracellular homeostasis $[9,10]$. Obesity and metabolic syndrome are intricately associated with T2DM [11] and are known to mediate its complications via oxidative stress $[5,12]$.

Several studies have shown serum MDA as a marker of lipid peroxidation in obese T2DM but hardly any study has established the association of lipid peroxidation and antioxidant status in nonobese T2DM subjects. Hence, the present study has attempted to explore the association between lipid peroxidation and antioxidant status in nonobese T2DM and further to correlate whether their significant association is putatively associated with the pathogenesis of nonobese T2DM.

\section{Materials and Methods}

2.1. Study Subjects. One hundred two (102) type 2 DM (T2DM) subjects (mean age: $53.1 \pm 7.86$ years) and ninety-five (95) nondiabetic healthy control (HC) subjects (mean age: $51.5 \pm 7.24$ years) were enrolled in this cross-sectional study. Presence of coronary artery disease (CAD) or strong family history of CAD, type $1 \mathrm{DM}$, hypertension, peripheral vascular disease, cancer, recent acute infection, thrombotic event, urinary microalbumin $>300 \mathrm{mg} /$ day, prediabetes (fasting blood glucose $>100 \mathrm{mg} / \mathrm{dL}$ but $<126 \mathrm{mg} / \mathrm{dL}$ and postprandial blood glucose $>140 \mathrm{mg} / \mathrm{dL}$ but $<200 \mathrm{mg} / \mathrm{dL}$ ), and neurological disorders was considered as exclusion criteria in this study.

The samples were obtained from the Outpatient Department of Endocrinology, Institute of Post Graduate Medical Education and Research, Kolkata. Written informed consent was collected from each patient and control subject after full explanation of the study. The study was approved by ethical committee of the institute according to the Declaration of Helsinki. Age, sex, and blood pressure were matched within the study groups. None of the T2DM subjects were on insulin treatment during the study period. There was no significant statistical difference in age, sex distribution, BMI, and blood pressure in nonobese T2DM patients and $\mathrm{HC}$ individuals. Fasting and postprandial (PP) plasma glucose (blood samples were drawn after $2 \mathrm{hrs}$ of $75 \mathrm{gm}$ postload glucose) and $\mathrm{HbA}_{1} \mathrm{c} \%$ were elevated significantly among $\mathrm{DM}$ subjects compared to HC individuals $(P<0.001)$ (Table 1$)$.

2.2. Sample Collection and Processing. The study subjects were advised to be in 12-hour strict fasting state, after which $15 \mathrm{~mL}$ venous blood samples were drawn. $10 \mathrm{~mL}$ blood sample was collected in heparinized tube for PBMC isolation and $5 \mathrm{~mL}$ sample was taken to obtain plasma and serum in cryovials for MDA and thiol assays.
TABLE 1: Clinical characteristics of healthy controls (HC) and diabetic subjects (DM).

\begin{tabular}{lccc}
\hline & Control $(n=95)$ & DM $(n=102)$ & $P$ value \\
\hline Age (in years) & $51.5 \pm 7.24$ & $53.1 \pm 7.86$ & 0.303 \\
Sex $(\mathrm{M} / \mathrm{F})$ & $49 / 46$ & $54 / 48$ & 0.887 \\
BMI $\left(\mathrm{kg} / \mathrm{m}^{2}\right)$ & $23.20 \pm 1.69$ & $22.75 \pm 1.67$ & 0.061 \\
Duration of diabetes & - & $17.9 \pm 5.76$ & - \\
BP-systolic $(\mathrm{mm} \mathrm{Hg})$ & $127.8 \pm 8.4$ & $128.2 \pm 7.9$ & 0.201 \\
BP-diastolic $(\mathrm{mm} \mathrm{Hg})$ & $81.4 \pm 6.95$ & $82.2 \pm 6.5$ & 0.146 \\
FPG $(\mathrm{mg} / \mathrm{dL})$ & $80.94 \pm 8.5$ & $167.5 \pm 60.3^{*}$ & $<0.001$ \\
PPPG $(\mathrm{mg} / \mathrm{dL})$ & $114.3 \pm 9.9$ & $238 \pm 27.2^{*}$ & $<0.001$ \\
HbA $_{1}$ c $(\%)$ & $4.80 \pm 0.43$ & $7.48 \pm 1.03^{*}$ & $<0.001$ \\
\hline
\end{tabular}

FBG, fasting blood glucose; BP, blood pressure; PPPG, postprandial blood glucose. Age, $\mathrm{BMI}, \mathrm{HbA}_{1} \mathrm{c}$ scores, and serum levels of biochemical parameters were expressed as the mean $\pm \mathrm{SD}$. Statistically significant, ${ }^{*} P<0.001$.

Mononuclear cells from peripheral whole blood were obtained from $10 \mathrm{~mL}$ heparinized blood by using Histopaque 1077 separating media (Sigma Aldrich, USA) density gradient for 40 minutes at 1300 r.p.m and $20^{\circ} \mathrm{C}$ temperature. PBMCs were further subjected to centrifuge at 1500 r.p.m for 10 minutes and washed with 1X PBS (pH 7.2) two times. $5 \times$ $10^{5}$ and $5 \times 10^{6}$ cells were pelleted into two different tubes and resuspended in IX PBS ( $\mathrm{pH}$ 7.2) for the estimation of intracellular thiol.

2.3. Measurement of Total Thiol. Total thiol or sulfhydryl ($\mathrm{SH}$ ) groups in PBMC and serum were measured spectrophotometrically by Ellman's method [13], modified by $\mathrm{Hu}$ [14]. $5 \times$ $10^{6}$ mononuclear cells from each sample were homogenized in $100 \mu \mathrm{L}$ cold buffer $(100 \mathrm{mM}$ Tris- $\mathrm{HCl}$ containing $1 \mathrm{mM}$ EDTA, pH 7.5) for the estimation of PBMC total thiol level. Cells were centrifuged at 10,000 r.p.m for 15 minutes at $4^{\circ} \mathrm{C}$ and cell lysate was taken and diluted appropriately in TrisEDTA buffer to make the final protein concentration of $50 \mu \mathrm{g} / \mu \mathrm{L} .20 \mu \mathrm{L}$ of diluted cell lysate was used for intracellular thiol assay and expressed as $\mu \mathrm{mol}$ of thiol $/ \mathrm{mg}$ of protein. Thiols present in the sample (serum/cell lysate) reacted with $5,5^{\prime}$ dithiobis-(2-nitrobenzoic acid) (DTNB) and formed highly colored anion. According to manual protocol, $25 \mu \mathrm{L}$ of fresh serum was mixed with $1 \mathrm{~mL}$ Tris-EDTA buffer $(0.25 \mathrm{mmol} / \mathrm{L}$ Tris base, $20 \mathrm{mmol} / \mathrm{L}$ EDTA, $\mathrm{pH}$ 8.2) and the absorbance (A1) was measured spectrophotometrically (Halo DB-20, Dynamica, USA) at $412 \mathrm{~nm}$ and in the next step $12.5 \mu \mathrm{L}$ of DTNB solution (10 mM in absolute methanol) was added into the solution. After 15-minute incubation in ambient temperature, the absorbance (A2) was read again at $412 \mathrm{~nm}$ together with a DTNB blank. The concentration of total thiol in serum and PBMC samples was determined from linear standard curve established by 0.2 to $1.6 \mathrm{mmol} / \mathrm{L}$ and 0.05 to $0.8 \mu \mathrm{mol} / \mathrm{L}$ of reduced glutathione as sulfhydryl group standard, respectively.

2.4. Measurement of MDA. Lipid peroxidation in serum was measured by MDA estimation as described previously [15]. Lipoproteins in serum were precipitated by adding $20 \%$ trichloroacetic acid and $8.1 \%$ sodium dodecyl sulphate. 


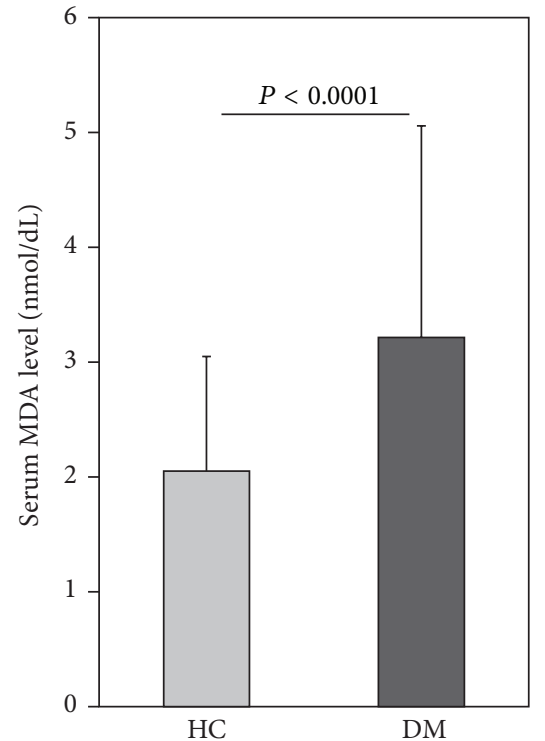

FIGURE 1: Serum malondialdehyde levels among healthy control and nonobese T2DM subjects. Values are expressed as the mean \pm SD. Statistically significant difference, $P<0.0001$, control versus T2DM.

Thereafter, $0.8 \%$ aqueous solution of thiobarbituric acid was added to this precipitate, mixed well, and finally heated at $95^{\circ} \mathrm{C}$ for 1 hour for coupling of lipid peroxide with thiobarbituric acid reagent. The resulting chromogen was extracted from the precipitate by adding n-butanol and pyridine mixture $(15: 1)$. The organic mixture was separated by centrifugation and the intensity of the organic layer was measured spectrophotometrically (Halo DB-20, Dynamica, Mayrwies, Salzburg, Austria) by using $530 \mathrm{~nm}$ filter against water blank. The concentration of MDA in serum was determined from linear standard curve established by 1 to $8 \mathrm{~nm}$ of 1,1,3,3-tetramethoxypropane.

2.5. Statistical Analysis. All variables were expressed as mean \pm SD (standard deviation). Mean values obtained from sample and control groups were compared by Student's unpaired two tailed $t$-test. Spearman's product moment correlation coefficient was used to find out the correlation between two variables. A value of $P<0.05$ was considered as statistically significant. All statistical analyses were performed by using GraphPad Prism software (version 5, 2007, San Diego, California, USA). Statistical analysis for sex distributions was evaluated by chi-square test by using statistical software STATA (version 8, Copyright 1984-2003, Stata Corporation, Texas, USA).

\section{Results}

Serum MDA level was found significantly higher among nonobese DM subjects compared to $\mathrm{HC}$ individuals ( $3.21 \pm$ 1.84 versus $2.05 \pm 0.99 \mathrm{nmol} / \mathrm{L} ; P<0.0001$ ) (Figure 1 ), whereas serum and PBMC total thiol levels were diminished significantly among DM subjects compared to HC subjects $(0.75 \pm 0.35$ versus $0.64 \pm 0.33 \mathrm{mmol} / \mathrm{L} ; P=0.0428,0.28 \pm 0.12$

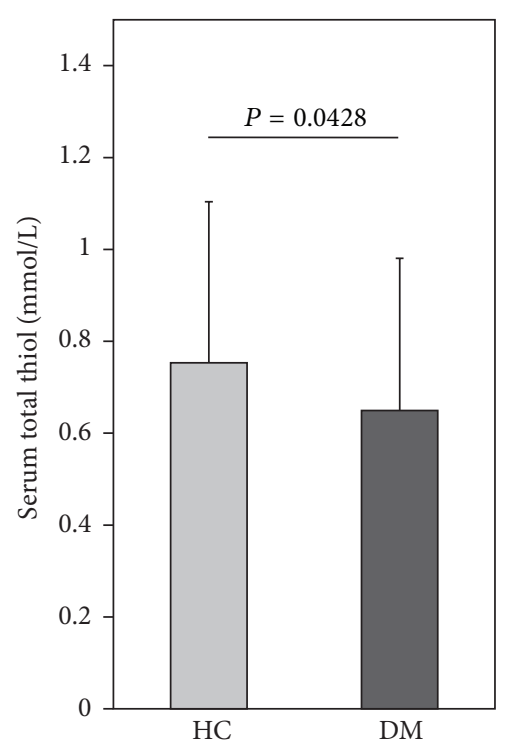

(a)

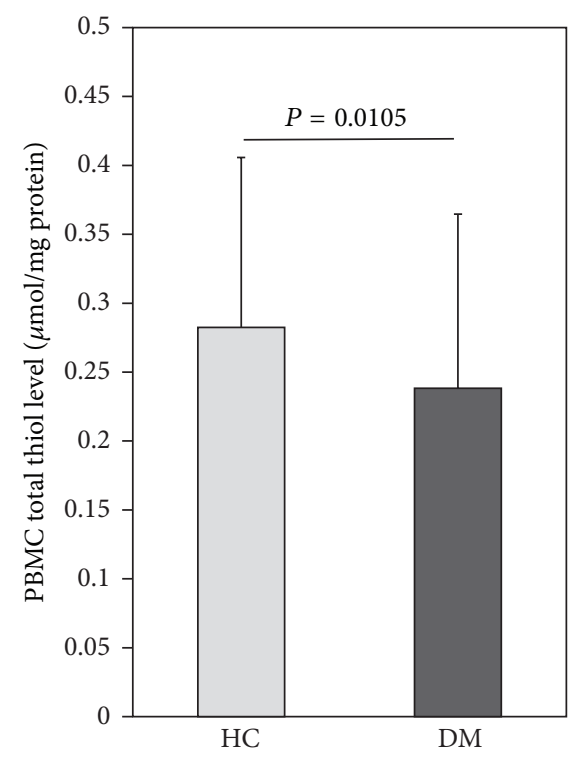

(b)

FIGURE 2: Serum total thiol (a) or PBMC total thiol (b) levels among healthy control and nonobese T2DM subjects. Values are expressed as the mean \pm SD. Statistically significant difference, $P<0.05$, control versus T2DM.

versus $0.23 \pm 0.11 \mu \mathrm{mol} / \mathrm{mg}$ of protein; $P=0.0105$, resp.) (Figure 2). Further, serum MDA level showed an inverse correlation with serum total thiol $(r=-0.3675 ; P=0.0001)$ level or PBMC thiol $(r=-0.3368 ; P=0.0005)$ level among DM subjects which were found to be statistically significant (Figure 3). However, the HC individuals did not have any significant correlation for serum total thiol $(r=0.1767 ; P=$ $0.0866)$ or PBMC thiol $(r=0.1153 ; P=0.2658)$ (Figure 3$)$.

\section{Discussion}

Over the past decade, there has been substantial interest on oxidative stress in the progression of type $2 \mathrm{DM}$. 


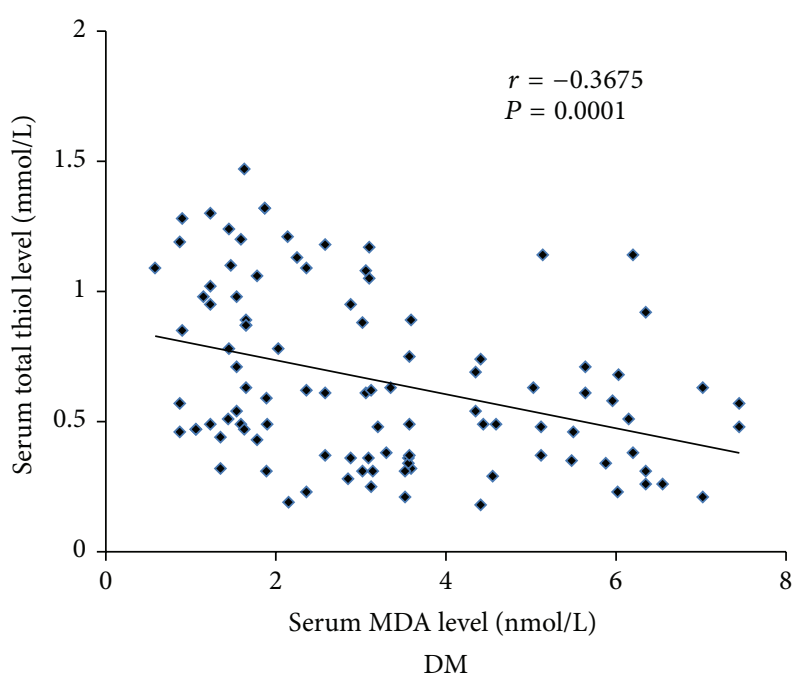

(a)

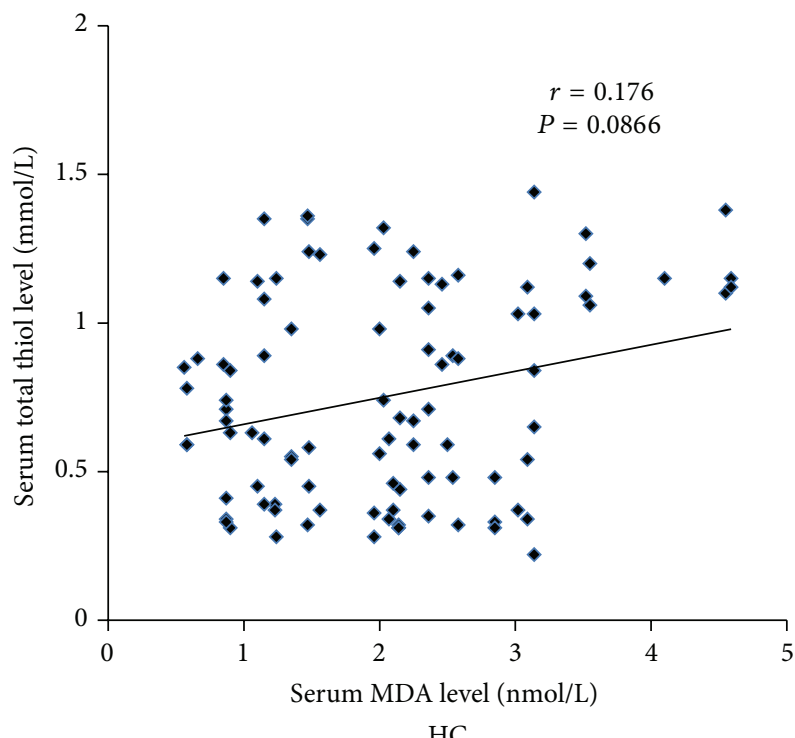

(c)

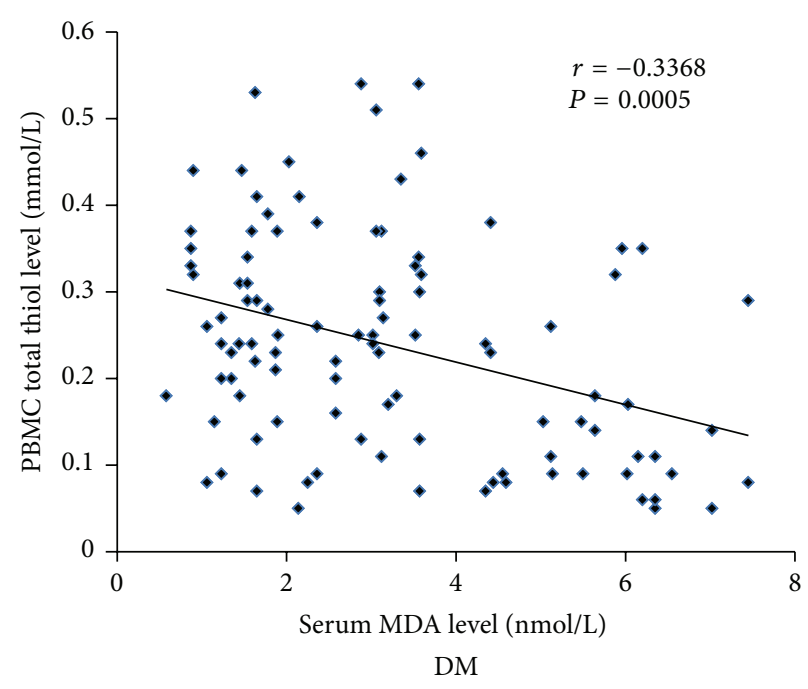

(b)

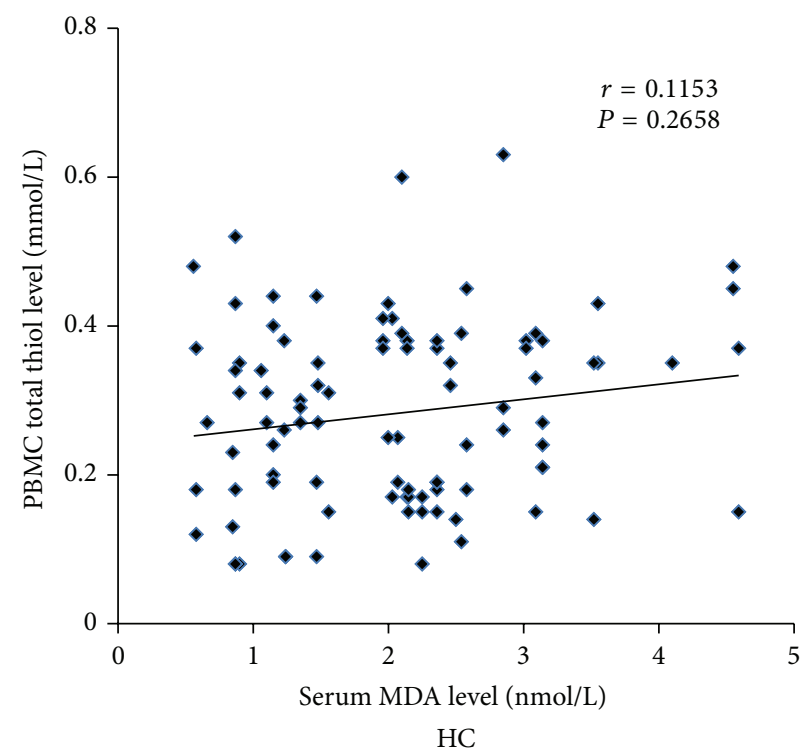

(d)

FIGURE 3: XY scatter plot represents correlation between serum MDA and serum total thiol or PBMC thiol levels among type 2 nonobese DM and healthy control individuals. Correlation coefficient $(r)$ represents the degree and nature of correlation between the MDA levels and serum total thiol or PBMC thiol levels in nonobese T2DM subjects and $\mathrm{HC}$ as described in text. A value of $P<0.05$ was considered statistically significant.

Hyperglycemic conditions are associated with elevated ROS production, predominantly through mitochondrial electron transport chain and NADPH oxidase [16-18]. Increased oxidative stress has been postulated to play a key role in the pathogenesis of DM associated complications like neuropathy, nephropathy, cardiomyopathy, and retinopathy [19-22]. Obesity itself being a chronic mild inflammatory condition and a key mediator of oxidative stress $[23,24]$ is closely linked to the etiopathogenesis of DM and its macro- and microvascular complications [11,25]. The possible sources of ROS include autoxidation of glucose, shifts in redox balance, decreased tissue concentration of glutathione and vitamin E, and impaired activity of superoxide dismutase (SOD) and catalase.
Oxidative stress has been widely established as a major contributory factor in the development as well as progression of diabetes and its complications. It has been suggested that insulin resistance may be accompanied by intracellular production of free radicals. Insulin has been found to increase the production of hydrogen peroxide in adipocytes cultured in vitro and this $\mathrm{H}_{2} \mathrm{O}_{2}$ has been shown to mimic the action of insulin. Thus, a vicious cycle between hyperinsulinemia and free radicals could be operating in the early stages of diabetes pathogenesis. Insulin resistance induced elevated plasma free radicals, in turn, may cause a deterioration of insulin action, with hyperglycemia being a contributory factor [26]. In studies relating to the pathogenesis of diabetic nephropathy, it has been shown that increased mitochondrial 
ROS induced by hyperglycemia leads to activation of the NF$\kappa \beta$ pathway. This may lead to a variety of effects including the increased production of the inducible form of cyclooxygenase (COX-2) and increased prostaglandin synthesis [27].

Other mechanisms by which increased oxidative stress is involved in the diabetic complications include activation of several transcription factors, protein kinase $C$, and advanced glycated end products (AGEs) [28]. It is, therefore, very important to explore the relationship between oxidative stress, diabetes, and its complications so that we can expand the treatment options. With obesity being a major contributory factor for increased ROS formation, we wanted to elucidate other possible factors leading to increased oxidative stress in nonobese type 2 DM population. Very few studies in the past have shown the association of oxidative stress markers in nonobese T2DM subjects $[12,29]$. In this study, we have taken nonobese T2DM patients to eliminate obesity induced oxidative stress but yet found surprising results. In the present study, we have found a significant elevation of serum MDA, an indicator of lipid peroxidation, in nonobese T2DM subjects as compared to HC subjects (Figure 1). This interesting finding is supported by Ajala et al. where MDA has been measured in plasma of 31 noncontrolled T2DM nonobese women [29]. Several studies indicate that elevated level of serum MDA among DM subjects speculates a close association of oxidative stress with the occurrence of diabetes [30, 31]. Total thiol pool constitutes majority portion of the total body antioxidants and plays major role in defense against $\operatorname{ROS}[8,32]$. Our study has shown that serum thiol levels are significantly lower in nonobese diabetic subjects compared to controls (Figure 2). This means that, apart from obesity, there are other factors and pathways that lead to oxidative stress and depletion of antioxidant pool in nonobese T2DM which needs to be explored further.

Even though our study on altered serum MDA and thiols in nonobese T2DM is interesting, several limitations in our study need to be mentioned which include nonestimation of MDA in erythrocytes and serum insulin, known to be related in this context. Prediabetic nonobese subjects were also not included in this study where serum MDA might be an alarming factor in the pathogenesis of T2DM subjects. Another limitation of this study is the nonassessment of pancreatic islet cell autoantibodies (especially GAD 65) which would have thrown light on the status of antioxidant pool in the presence and absence of the autoantibodies. Another important limitation of the present study as well as other case control studies is its relation to the medication therapy of T2DM subjects. All the T2DM subjects in this study were on sulphonylureas or metformin either as monotherapy or in combination of both drugs. Further, many of these patients were also given intermittent or long term courses of vitamins and antihypertensive drugs. It is rather unknown whether these drugs have confounded our study results to some extent, but despite such differences in the drug history, it has been possible to obtain clear changes in the serum MDA level in nonobese T2DM subjects with respect to controls which is in good correlation with total serum thiol and total PBMC thiol levels. Decreased levels of total thiols in PBMC and serum of DM subjects could be due to increased oxidation of - $\mathrm{SH}$ groups due to oxidative stress, which was reflected by higher serum MDA level in nonobese T2DM subjects. This deprived antioxidant state among T2DM patients may explain the increased susceptibility of microvasculature to oxidative injury in poorly controlled patients of T2DM. Further significant inverse correlation of serum MDA level and serum total thiol level among DM subjects suggests a close association of increased oxidative stress with decreased antioxidant status in nonobese diabetes. It may also be concluded that serum MDA may be used as a potential biomarker in nonobese T2DM subjects but, nevertheless, a larger cross-sectional study is essential to conclude the fact.

\section{Conflict of Interests}

The authors declare that there is no conflict of interests regarding the publication of this paper.

\section{Acknowledgments}

The authors acknowledge Professor Pradip Kumar Mitra, Director, IPGMER, for financial assistance and encouragement and Professor Sasanka Chakrabarti, Head of the Department of Biochemistry, IPGMER, for his advice and guidance. They thank "The West Bengal University of Health Sciences" for help and assistance. The authors also acknowledge Dr. Soumyabrata Munshi for his suggestions and constructive criticisms during preparation of this paper.

\section{References}

[1] H. King, R. E. Aubert, and W. H. Herman, "Global burden of diabetes, 1995-2025: prevalence, numerical estimates, and projections," Diabetes Care, vol. 21, no. 9, pp. 1414-1431, 1998.

[2] H. A. Keenan, T. Costacou, J. K. Sun et al., "Clinical factors associated with resistance to microvascular complications in diabetic patients of extreme disease duration: the 50-year medalist study," Diabetes Care, vol. 30, no. 8, pp. 1995-1997, 2007.

[3] S. E. Kahn, "The relative contributions of insulin resistance and beta-cell dysfunction to the pathophysiology of Type 2 diabetes," Diabetologia, vol. 46, no. 1, pp. 3-19, 2003.

[4] R. A. DeFronzo, "Pathogenesis of type 2 diabetes mellitus," The Medical Clinics of North America, vol. 88, no. 4, pp. 787-835, 2004.

[5] S. D. M. Bandeira, L. J. S. da Fonseca, G. D. S. Guedes, L. A. Rabelo, M. O. F. Goulart, and S. M. L. Vasconcelos, "Oxidative stress as an underlying contributor in the development of chronic complications in diabetes mellitus," International Journal of Molecular Sciences, vol. 14, no. 2, pp. 3265-3284, 2013.

[6] G. Basta, G. Lazzerini, M. Massaro et al., "Advanced glycation end products activate endothelium through signal-transduction receptor RAGE: a mechanism for amplification of inflammatory responses," Circulation, vol. 105, no. 7, pp. 816-822, 2002.

[7] R. A. Kowluru and P.-S. Chan, "Oxidative stress and diabetic retinopathy," Experimental Diabesity Research, vol. 2007, Article ID 43603, 12 pages, 2007.

[8] R. Mancino, D. di Pierro, C. Varesi et al., "Lipid peroxidation and total antioxidant capacity in vitreous, aqueous humor, and blood samples from patients with diabetic retinopathy," Molecular Vision, vol. 17, pp. 1298-1304, 2011. 
[9] R. Rossi, D. Giustarini, A. Milzani, and I. Dalle-Donne, "Cysteinylation and homocysteinylation of plasma protein thiols during ageing of healthy human beings," Journal of Cellular and Molecular Medicine, vol. 13, no. 9B, pp. 3131-3140, 2009.

[10] G. Baskol, K. Gumus, A. Oner, H. Arda, and S. Karakucuk, "The role of advanced oxidation protein products and total thiols in diabetic retinopathy," European Journal of Ophthalmology, vol. 18, no. 5, pp. 792-798, 2008.

[11] N. Esser, S. Legrand-Poels, J. Piette, A. J. Scheen, and N. Paquot, "Inflammation as a link between obesity, metabolic syndrome and type 2 diabetes," Diabetes Research and Clinical Practice, vol. 14, pp. 187-189, 2014.

[12] R. Goyal, M. Singhai, and A. F. Faizy, "Glutathione peroxidase activity in obese and non-obese diabetic patients and role of hyperglycemia in oxidative stress," Journal of Midlife Health, vol. 2, no. 2, pp. 72-76, 2011.

[13] G. L. Ellman, "Tissue sulfhydryl groups," Archives of Biochemistry and Biophysics, vol. 82, no. 1, pp. 70-77, 1959.

[14] M.-L. Hu, "Measurement of protein thiol groups and glutathione in plasma," Methods in Enzymology, vol. 233, pp. 380385, 1994.

[15] K. Satoh, "Serum lipid peroxide in cerebrovascular disorders determined by a new colorimetric method," Clinica Chimica Acta, vol. 90, no. 1, pp. 37-43, 1978.

[16] H. Izuta, N. Matsunaga, M. Shimazawa, T. Sugiyama, T. Ikeda, and H. Hara, "Proliferative diabetic retinopathy and relations among antioxidant activity, oxidative stress, and VEGF in the vitreous body," Molecular Vision, vol. 16, pp. 130-136, 2010.

[17] D. Dymkowska, B. Drabarek, P. Podszywałow-Bartnicka, J. Szczepanowska, and K. Zabłocki, "Hyperglycaemia modifies energy metabolism and reactive oxygen species formation in endothelial cells in vitro," Archives of Biochemistry and Biophysics, vol. 542, pp. 7-13, 2014.

[18] R. Ray and A. M. Shah, "NADPH oxidase and endothelial cell function," Clinical Science, vol. 109, no. 3, pp. 217-226, 2005.

[19] J. W. Baynes, "Role of oxidative stress in development of complications in diabetes," Diabetes, vol. 40, no. 4, pp. 405-412, 1991.

[20] F. Giacco and M. Brownlee, "Oxidative stress and diabetic complications," Circulation Research, vol. 107, no. 9, pp. 10581070, 2010.

[21] M. Williams, R. E. Hogg, and U. Chakravarthy, "Antioxidants and diabetic retinopathy," Current Diabetes Reports, vol. 13, no. 4, pp. 481-487, 2013.

[22] S. A. Madsen-Bouterse and R. A. Kowluru, "Oxidative stress and diabetic retinopathy: pathophysiological mechanisms and treatment perspectives," Reviews in Endocrine and Metabolic Disorders, vol. 9, no. 4, pp. 315-327, 2008.

[23] A. I. Rupérez, A. Gil, and C. M. Aguilera, "Genetics of oxidative stress in obesity," International Journal of Molecular Sciences, vol. 15, no. 2, pp. 3118-3144, 2014.

[24] S. Gao, H. K. Roberts, and X. Wang, "Cross tissue traitpathway network reveals the importance of oxidative stress and inflammation pathways in obesity-induced diabetes in mouse," PLoS ONE, vol. 7, no. 9, Article ID e44544, 2012.

[25] D. Pitocco, M. Tesauro, R. Alessandro, G. Ghirlanda, and C. Cardillo, "Oxidative stress in diabetes: implications for vascular and other complications," International Journal of Molecular Sciences, vol. 14, no. 11, pp. 21525-21550, 2013.

[26] A. Ceriello, "Oxidative stress and glycemic regulation," Metabolism: Clinical and Experimental, vol. 49, no. 2, pp. 27-29, 2000.
[27] S. Kiritoshi, T. Nishikawa, K. Sonoda et al., "Reactive oxygen species from mitochondria induce cyclooxygenase-2 gene expression in human mesangial cells: potential role in diabetic nephropathy," Diabetes, vol. 52, no. 10, pp. 2570-2577, 2003.

[28] A. C. Maritim, R. A. Sanders, and J. B. Watkins III, "Diabetes, oxidative stress, and antioxidants: a review," Journal of Biochemical and Molecular Toxicology, vol. 17, no. 1, pp. 24-38, 2003.

[29] M. O. Ajala, P. S. Ogunro, S. E. Idogun, and O. Osundeko, "Relationship between plasma antioxidant status and leptin in controlled and non-controlled type 2 diabetic non-obese women," International Journal of Endocrinology \& Metabolism, vol. 7, no. 4, pp. 214-221, 2009.

[30] D. A. Slatter, C. H. Bolton, and A. J. Bailey, "The importance of lipid-derived malondialdehyde in diabetes mellitus," Diabetologia, vol. 43, no. 5, pp. 550-557, 2000.

[31] M. Kaefer, J. A. M. de Carvalho, S. J. Piva et al., "Plasma malondialdehyde levels and risk factors for the development of chronic complications in type 2 diabetic patients on insulin therapy," Clinical Laboratory, vol. 58, no. 9-10, pp. 973-978, 2012.

[32] H. Paşaoglu, B. Sancak, and N. Bukan, "Lipid peroxidation and resistance to oxidation in patients with type 2 diabetes mellitus," Tohoku Journal of Experimental Medicine, vol. 203, no. 3, pp. 211218, 2004. 


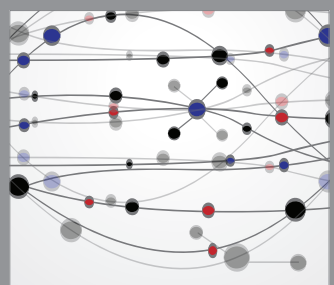

The Scientific World Journal
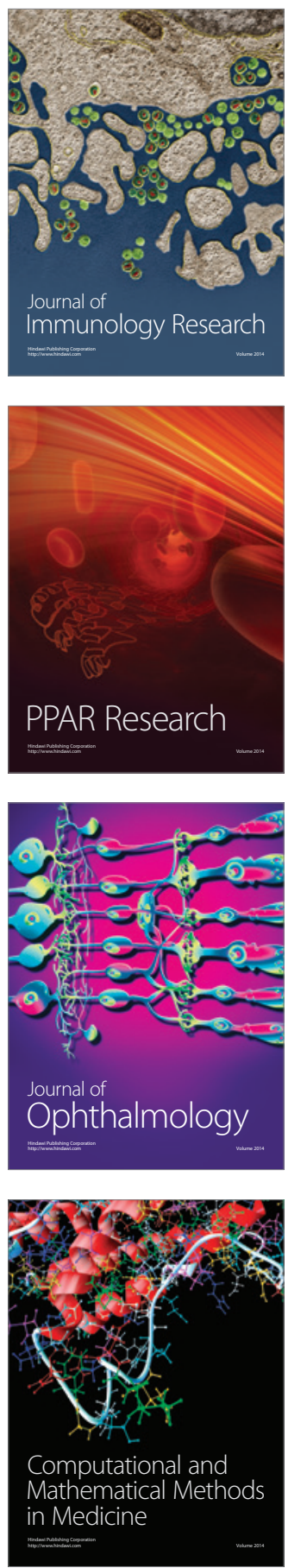

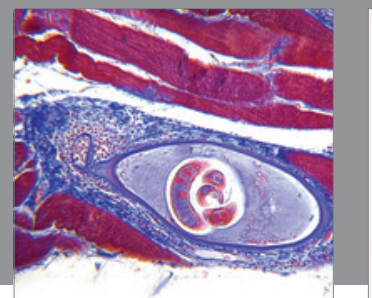

Gastroenterology

Research and Practice
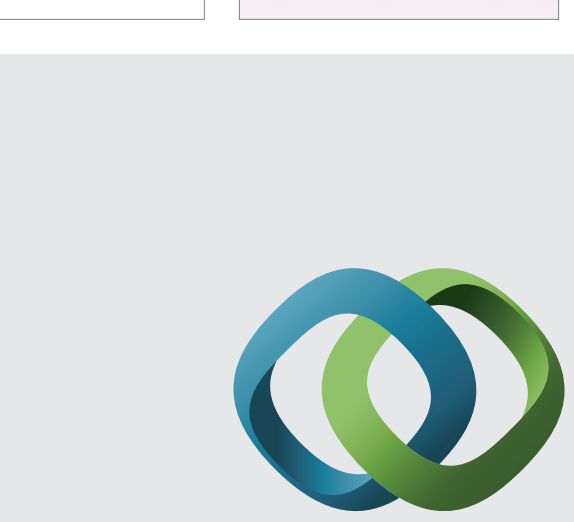

\section{Hindawi}

Submit your manuscripts at

http://www.hindawi.com
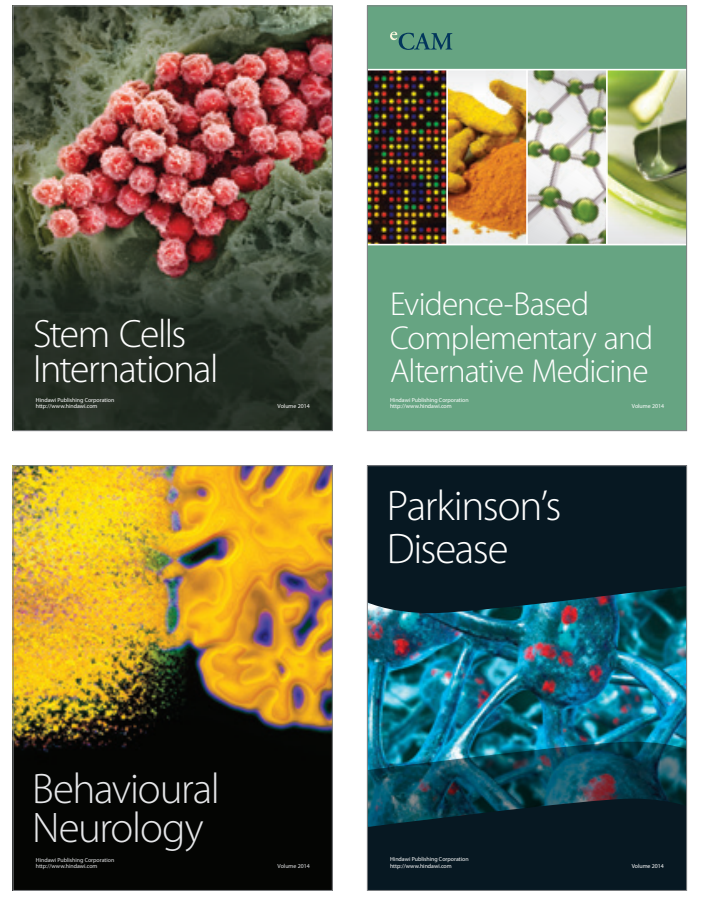
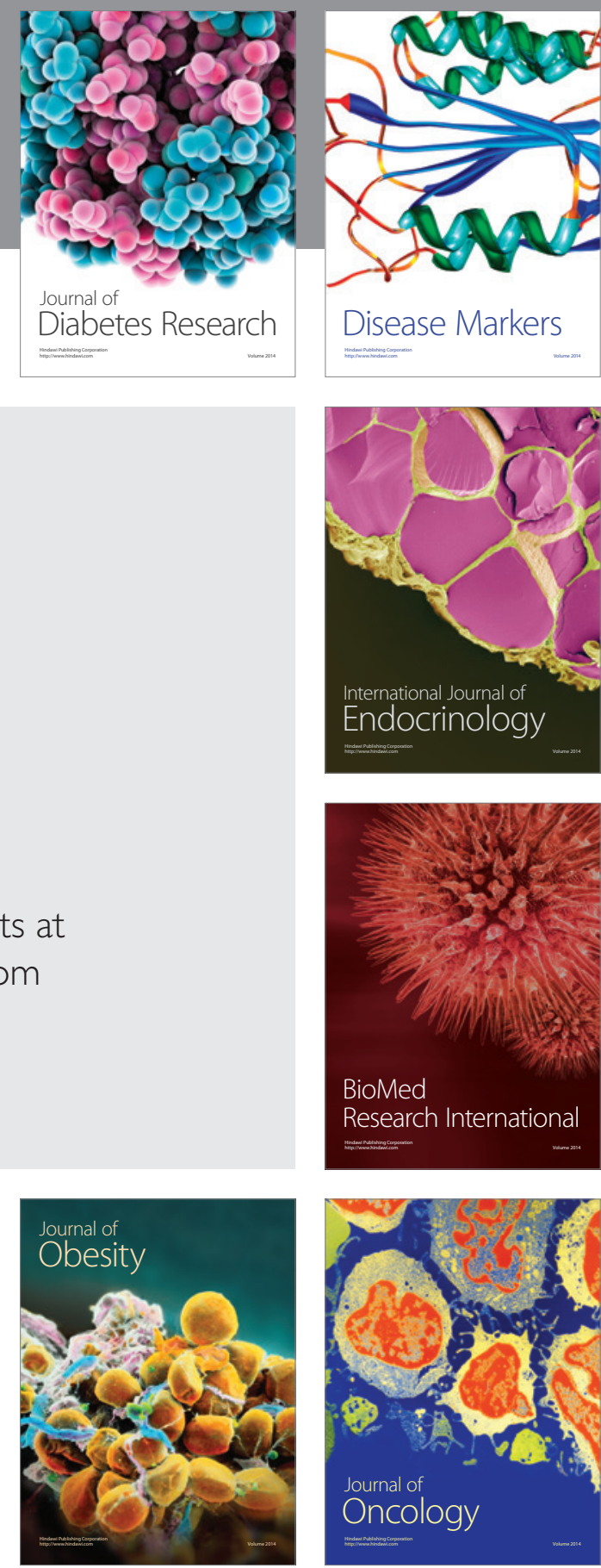

Disease Markers
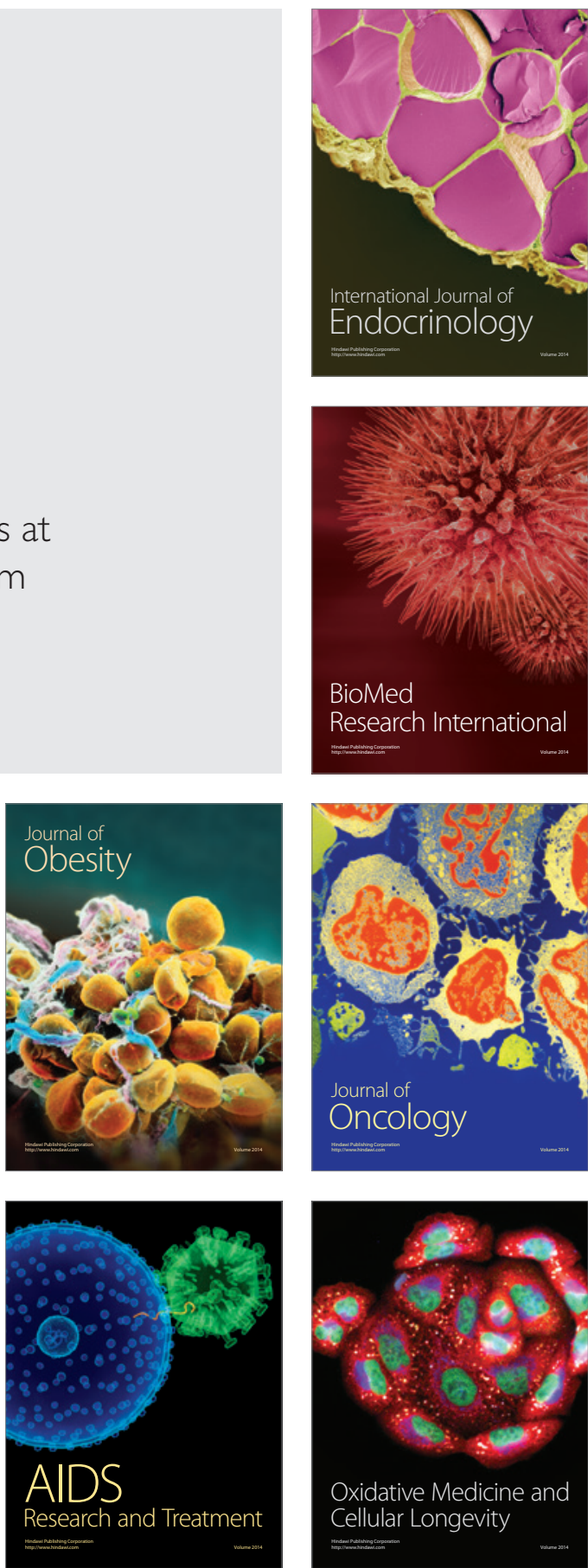